\title{
Profili storici
}

not peer reviewed

\section{Ricordo di Pietro Bruno Celico (1941-2015) \\ In commemoration of Pietro Bruno Celico (1941-2015)}

\author{
Anna Rosa Scalise ${ }^{a}$ \\ ${ }^{a)}$ Già ISPRA- Istituto Superiore per la Ricerca e Protezione Ambientale, Roma - e-mail: arscalise@gmail.com
}

Keywords: history of geosciences, Pietro Bruno Celico, Southern Apennines, structural hydrogeology.

Parole chiave: Appennino Meridionale, idrogeologia strutturale, Pietro Bruno Celico, storia delle geoscienze.

Nel 2021 Pietro Bruno Celico avrebbe compiuto 80 anni; la rubrica "profili storici" vuole onorare questa ricorrenza con un ricordo del personaggio, figura centrale dell'idrogeologia italiana.

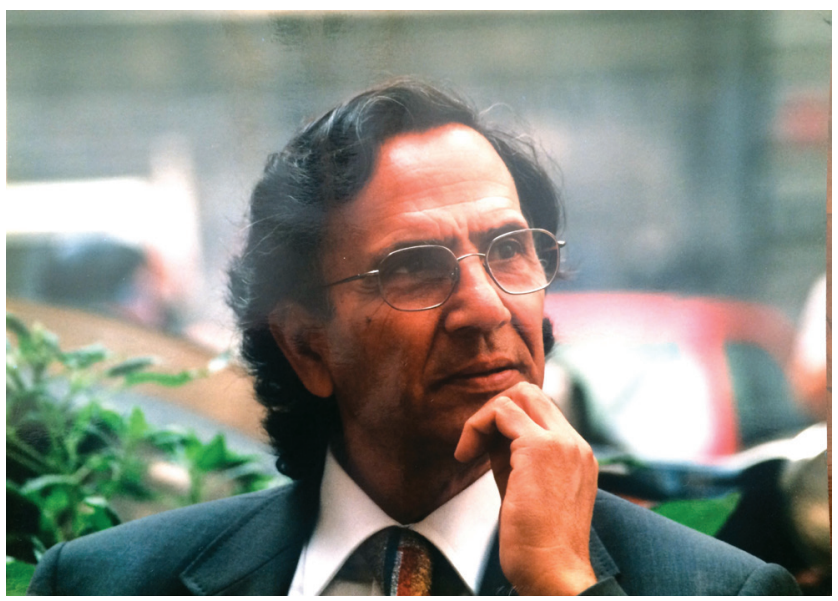

La Sua figura di "padre fondatore" dell'idrogeologia italiana è stata ricordata, in una giornata di studio che si è tenuta il 22 gennaio 2016, dall'allieva Silvia Fabbrocino, che ha sottolineato come, sul piano strettamente scientifico: "le sue monografie ed opere cartografiche hanno rappresentato, e tuttora rappresentano, il riferimento fondamentale per la conoscenza idrogeologica del territorio italiano e per la gestione quantitativa e qualitativa delle risorse idriche sotterranee. Basti ricordare la Memoria "Considerazioni sull'idrogeologia di alcune zone dell'Italia centro-meridionale alla luce dei risultati di recenti indagini geognostiche», pubblicata nel 1979, che ha contribuito fortemente all'avanzamento delle conoscenze sulla circolazione idrica basale degli acquiferi carbonatici, divenendo il punto di partenza per la progettazione dei più grandi sistemi acquedottistici ed infrastrutturali".

Nato a Cosenza nel 1941, Celico si laureò in Scienze Geologiche nel 1975, con il massimo dei voti e la lode, presso la facoltà di Scienze dell'Università di Napoli; dopo la laurea prestò la propria opera di geologo presso la Cassa per il Mezzogiorno, svolgendo un'intensa attività per ricerca, captazione ed utilizzazione ottimale delle risorse idriche sotterranee, soprattutto nell'ambito del "Progetto Speciale per il reperimento e l'utilizzazione razionale delle risorse idriche superficiali e sotterranee delle Regioni Marche, Abruzzo, Molise, Lazio e Campania (P.S. 29)": attività che lo vide coinvolto nella programmazione, progettazione, direzione tecnica delle ricerche e lavori di captazione di acque sotterranee, soprattutto di acquiferi carbonatici. E non solo: suoi campi d'azione furono pure indagini finalizzate alla progettazione di importanti dighe; studio di tracciati e siti acquedottistici sia sotterranei che seminterrati; studio della stabilità dei versanti di aree interessate da importanti opere ingegneristiche quali dighe, acquedotti, ecc.

In contemporanea, egli svolse un'intensa attività di ricerca scientifica, parzialmente sintetizzata in oltre 230 pubblicazioni su periodici nazionali ed internazionali: numerosi sono gli elementi di assoluta originalità per la definizione sia dell'approccio metodologico alla ricostruzione dell'idrodinamica sotterranea in acquiferi complessi, con particolare riferimento a quelli carbonatici, che della vulnerabilità degli stessi all' inquinamento e della salvaguardia quali-quantitativa e della gestione ottimale delle risorse idriche sotterranee, comprese quelle minerali.

Nel 1983 pubblicò "Idrogeologia dei massicci carbonatici, delle piane quaternarie e delle aree vulcaniche dell'Italia centro-meridionale (Marche e Lazio meridionali, Abruzzo, Molise e Campania)", Quaderno 4/2 della Cassa per il Mezzogiorno, che è stato - e continua ad essere - una sorta di 'vangelo' per quanti si siano avvicinati e si avvicinano allo studio completo ed integrato di quelle aree. Tale opera ha rappresentato una svolta significativa per la conoscenza dei fattori condizionanti la circolazione idrica basale degli acquiferi carbonatici ed i limiti dei bacini sotterranei, con vari elementi di novità nella definizione del comportamento idrogeologico degli elementi strutturali e nella individuazione di serbatoi sotterranei funzionanti "in serie".

Ma l'intensa attività scientifica è testimoniata anche dalla notevolissima produzione cartografica: la Carta Idrogeologica d'Italia alla scala 1:500.000, stampata a cura della "Commission des Communautés européennes"; la Carta Idrogeologica della 
Campania alla scala 1:200.000; la Carta Idrogeologica dell'Italia centro-meridionale alla scala 1:400.000; la Carta Idrogeologica dell'Italia Meridionale alla scala 1:250.000; il F.186 "S. Angelo dei Lombardi" della Carta Idrogeologica alla scala 1:100.000; la Carta Idrogeologica della provincia di Napoli alla scala 1:50.000 e la Carta Idrogeologica della provincia di Avellino alla scala 1:100.000.

Partecipò attivamente a Progetti Finalizzati del CNR, come quello relativo alla Geotermia, con particolare riferimento alla geochimica delle acque sotterranee: in un tale ambito rientrano le ricerche relative alle caratteristiche idrogeologiche della zona flegrea, con specifica attenzione allo studio del bilancio di massa e di energia ed al quello degli effetti delle iniezioni di fluido nel sottosuolo. Altri momenti importanti furono la partecipazione ai lavori di due Commissioni tecnicoscientifiche, istituite dal Ministero dei Lavori Pubblici, la prima incaricata dello studio della regimazione delle acque e della sistemazione idraulica del bacino del fiume Liri e l'altra dell'utilizzazione ottimale e di una maggiore e migliore protezione delle acque termo-minerali di Castellammare di Stabia, alimentate da un importante acquifero carbonatico.

Nel 1986, pubblicò il primo volume del celebre manuale "Prospezioni idrogeologiche" e, nel 1988, il secondo. Si tratta di testi completi e molto ricchi di elementi per lo studio dell'idrogeologia a livello sia specialistico che di corso universitario (ed è questa una delle loro peculiarità), oltre che esauriente guida pratica all'esecuzione di indagini idrogeologiche finalizzate a captazione e gestione ottimale delle risorse idriche.

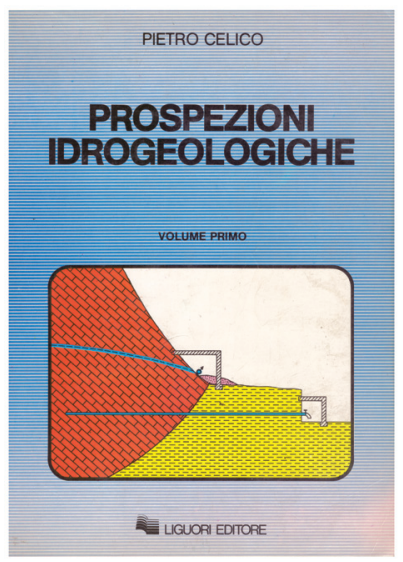

Nel 1989 fu nominato componente del "Gruppo di lavoro per la normativa sulla cartografia idrogeologica” del Servizio Geologico d'Italia, incaricato di elaborare linee guida per la redazione della cartografia idrogeologica alla scala 1:50.000.

Negli anni novanta, nell'ambito del GNDCI del CNR, fu responsabile dell'Unità Operativa per lo studio degli aspetti idrogeologici connessi con i problemi di prevenzione e previsione delle piene, nonchè dell'idrodinamica sotterranea degli acquiferi carbonatici e della vulnerabilità all'inquinamento di quelli complessi. Nel 1993 fu nominato membro delle Commissioni di Studio relative al "Corso di aggiornamento e di preparazione agli esami di stato" ed
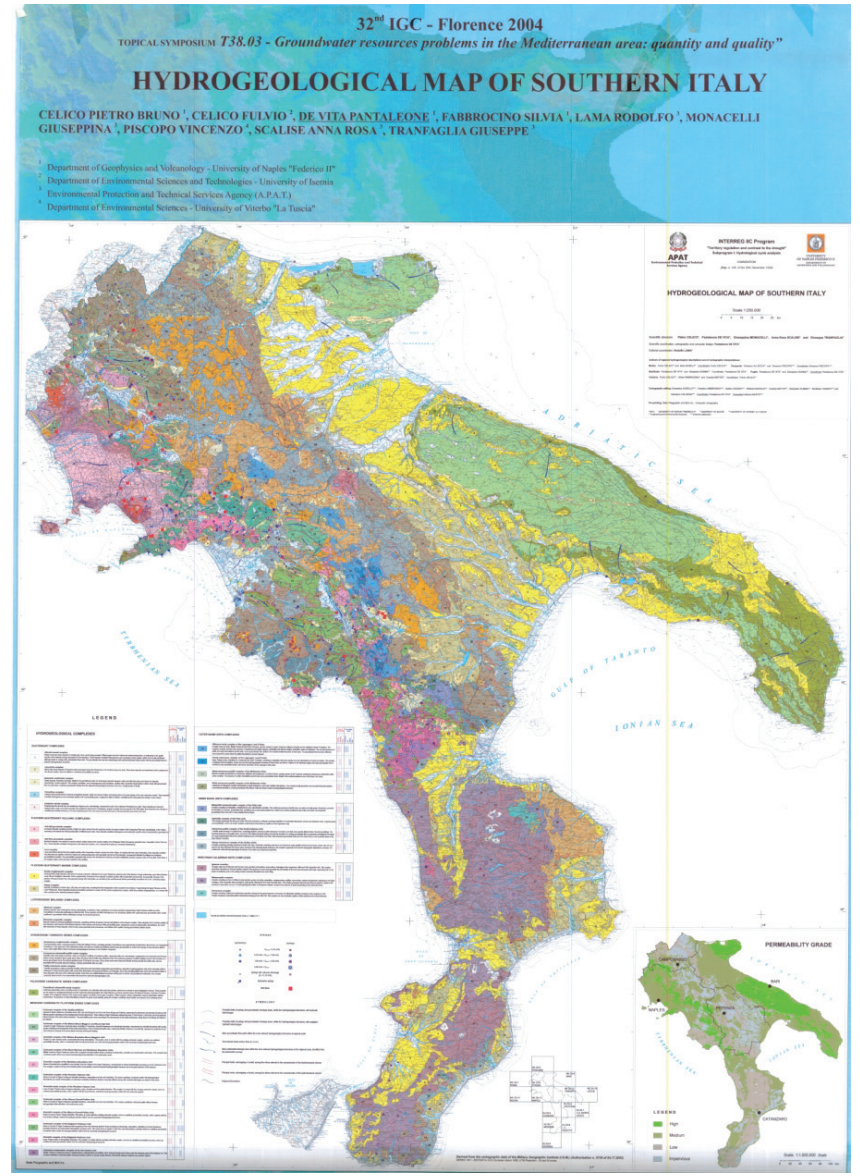

al "Corso di prospezioni dirette e indirette e prove relative alle ricerche idriche", organizzati dall'Ordine dei Geologi della Regione Campania; nell'anno successivo tenne lezioni di Idrogeologia nell'ambito di Corsi di Aggiornamento, organizzati dall'Ordine Regionale di Geologi della Calabria.

Nel 1996 venne chiamato a far parte del Gruppo di Lavoro per la Salvaguardia Ambientale del Torrente Solofrana e, nel 1997, nell'ambito delle iniziative del CUGRI dell'Università di Salerno, fu responsabile del settore Falde Acquifere. Nello stesso anno, coordinò il programma relativo alle Carte idrogeologiche e della vulnerabilità all'inquinamento degli acquiferi della Regione Calabria. Risale all'anno successivo la nomina a membro di una Commissione per l'esame della bozza del testo unico sulla tutela delle acque dall'inquinamento.

Tra gli impegni di carattere professionale, è da sottolineare come, negli ultimi anni, si dedicò principalmente ai Piani di Tutela delle Acque, nello specifico della Regione Abruzzo e della Regione Campania (di quest'ultimo fu Coordinatore Generale e Responsabile Scientifico).

Concorse, nel 2000, a fondare l'Associazione Italiana di Geologia Applicata (AlGA), della quale fu membro del Consiglio Direttivo.

Intensa l'attività didattica, iniziata nel 1975 presso la Facoltà di Ingegneria dell'Università di Napoli con il Corso di Idraulica per Ingegneria Civile al quale, nel 1977, si aggiunse quello di Geochimica delle acque. Presso l'Università di Palermo tenne, nell'anno accademico 1984/85, il corso di 
"Metodi di prospezione idrogeologica in acquiferi carbonatici. A partire dall'Anno Accademico 1987/1988 divenne Professore Associato di Geologia Applicata presso la Facoltà di Scienze MM. FF. e NN. dell'Università Federico II.

Nell'Anno Accademico 1990/1991 divenuto Professore Ordinario di Geologia Applicata, ricoprì la Cattedra di Idrogeologia che tenne sino al collocamento a riposo, avvenuto nel 2011.

Di vari momenti di quel periodo si menzionano pure il corso di Idrogeologia (Facoltà di Ingegneria dell'Università di Salerno) e quello di Idrogeologia ed Idrogeologia Applicata, svolto presso l'Università del Sannio e quella della Calabria.

Pietro Bruno Celico aveva una personalità straordinaria fuori dal comune, emergeva per la sua totale disponibilità, pari ad un carattere posato e un altissimo livello scientifico.

Nel corso della sua intensa attività didattica spiegava sempre, con estrema semplicità, le teorie dell'idrogeologia, accompagnandole con esempi pratici e concreti che agevolavano l'apprendimento dei suoi studenti: di sicuro, la sua principale dote.

Straordinaria la naturalezza con la quale era sempre pronto a dare una mano nel risolvere le questioni scientifiche e tecniche che di tanto in tanto gli venivano sottoposte: prezioso, un tale modo di essere, in occasione dell'emergenza rifiuti nella regione Campania, concretizzato in una collaborazione ed in una serie di suggerimenti dettati dalla sua altissima cultura e dalla vastissima esperienza professionale.

Tra i lavori scientifici svolti in collaborazione vorrei accennare alla Carta idrogeologica meridionale alla scala 1:250.000 e alle relative note illustrative e alla Sperimentazione delle Linee Guida della Cartografia Idrogeologica, nell'area dei Monti del Matese, della Piana di Boiano-Monte Totila e della Piana del F. Sordo.

Il ricordo di Pietro Bruno Celico è quello di un caro maestro ed un amico affettuoso da ricordare come una persona autentica ricca di veri sentimenti.

A ribattezzarlo "il Sommo" furono, intorno alla fine degli anni '80 dello scorso secolo, Mario Valletta ed una comune allieva. Il parimenti compianto Valletta lo ricordava come "uno dei pochissimi autentici Maestri che le Scienze della Terra abbiano avuto nell'ultimo cinquantennio, un ricercatore di razza e di altissimo profilo che ha contrassegnato tappe fondamentali nella evoluzione delle conoscenze idrogeologiche di larga parte del nostro Paese. Tappe raggiunte attraverso quello che è sempre stato, rimane e rimarrà, l'unico ed insostituibile approccio, vale a dire un'accurata indagine di campo, effettuata con mente libera da modelli da "dover dimostrare", anche forzando la realtà, come spessissimo è capitato: "mente et malleolo", insomma, secondo la perifrasi di Bruno D'Argenio del classico "mente et malleo". Tantissimi ricordi di quasi un quarantennio di amicizia fraterna dell'Uomo Pietro e della sua nobiltà d'animo e della sua straordinaria apertura mentale. E come non ricordare quanto si sia adoperato per contribuire, prima, alla fase organizzativa di un Convegno promosso, insieme al Servizio Geologico d'Italia, per ricordare Carlo Bergomi nel decennale della sua tragica scomparsa nei Monti del Matese e, poi, quale chairman di quella parte dei lavori dedicata all'idrogeologia, preoccupandosi pure di ottenere i necessari permessi per la visita, da Lui guidata, alle sorgenti Torano e Maretto e quanta cura e meticolosità riponesse in ogni dettaglio in particolar modo a quella relativa al settore meridionale dei Monti del Sannio".

I geologi ricordano Pietro Bruno Celico tra i padri fondatori dell'Idrogeologia, tutta la comunica scientifica e quella professionale ne ricordano la figura, l'esempio, gli insegnamenti e il contributo straordinario che rimarranno sempre vivi e presenti in maniera indelebile.

A noi non resta che ringraziare Pietro Bruno Celico del contributo che ha dato alla geologia e alla conoscenza idrogeologia del territorio italiano.

\section{Bibliografia}

Allocca V, Celico F, Celico PB, De Vita P, Fabbrocino S, Mattia C, Monacelli G, Musilli I, Piscopo V, Scalise AR, Summa G, Tranfaglia G (2009) La carta idrogeologica dell'Italia meridionale. Metodi ed analisi territoriali per l'identificazione e la caratterizzazione dei corpi idrici sotterranei (Direttiva 2000/60/CE). "L'ACQUA" Rivista bimestrale dell'ass. idrot. It. 4/2009 - Luglio-Agosto, pp. 21-32. ISSN 1125-1255.

APAT e Università degli Studi di Napoli Federico II (2005) Carta Idrogeologica dell'Italia meridionale - Scala 1:250.000 (Responsabili Scientifici Celico PB, De Vita P, Monacelli G, Scalise AR, Tranfaglia G), Istituto Poligrafico dello Stato. ISBN 88-448-0223-6 Program INTERREG IIC "Assetto del territorio e lotta contro la siccità" Sottoprogramma I: Analisi del ciclo idrologico.

APAT e Università degli Studi di Napoli Federico II (2005) Note illustrative alla Carta Idrogeologica meridionale. Allocca V, Celico F, Celico PB, De Vita P,Fabbrocino S, Mattia C, Monacelli G, Musilli I, Piscopo V, Scalise AR, Summa G, Tranfaglia G. Istituto Poligrafico e Zecca dello Stato ISBN 88-448-0215-5 p. 211, (3 tavole fuori testo). Program INTERREG IIC "Assetto del territorio e lotta contro la siccità" Sottoprogramma I: Analisi del ciclo idrologico

Budetta P, Celico PB, Corniello A, De Riso R, Ducci D, Nicotera P (1994) Carta idrogeologica della Campania alla scala 1:200.000. Memoria illustrativa, $4^{\circ}$ Convegno Internazionale Geoingegneria, Torino, 565-586

Celico PB (1979) Considerazioni sull'idrogeologia di alcune zone dell'Italia centro-meridionale alla luce dei risultati di recenti indagini geognostiche. Mem. e Note Ist.Geol.Appl., 15, pp.1-43, Napoli.

Celico PB (1983) Idrogeologia dei massicci carbonatici, delle piane quaternarie e delle aree vulcaniche dell'Italia centro-meridionale (Marche e Lazio meridionali, Abruzzo, Molise e Campania). Quad. Cassa per il Mezzogiorno, 4, 2,225 Roma.

Celico PB (1983) Carta Idrogeologica dell'Italia centro-meridionale (Marche e Lazio meridionali, Abruzzo, Molise e Campania) alla scala 1:400.000. Cassa per il Mezzogiorno, Grafiche Magliana, Roma.

Celico PB (2009) Ordinario di Idrogeologia, Università degli Studi "Federico II" di Napoli. Curriculum, Napoli.

De Vita P, Allocca V, Celico F, Fabbrocino S, Cesaria M, Monacelli G, Musilli I, Piscopo V, Scalise AR, Summa G, Tranfaglia G, Celico PB (2018) Hydrogeology of continental southern Italy Journal of Maps, 14.2, 230-241.

Scalise A. R., Valletta M. (2018) Pietro Bruno Celico: Il "Sommo". www.geoitaliani.it. 